\title{
Aplicación de la espectrometría infrarroja al análisis químico de los bienes culturales
}

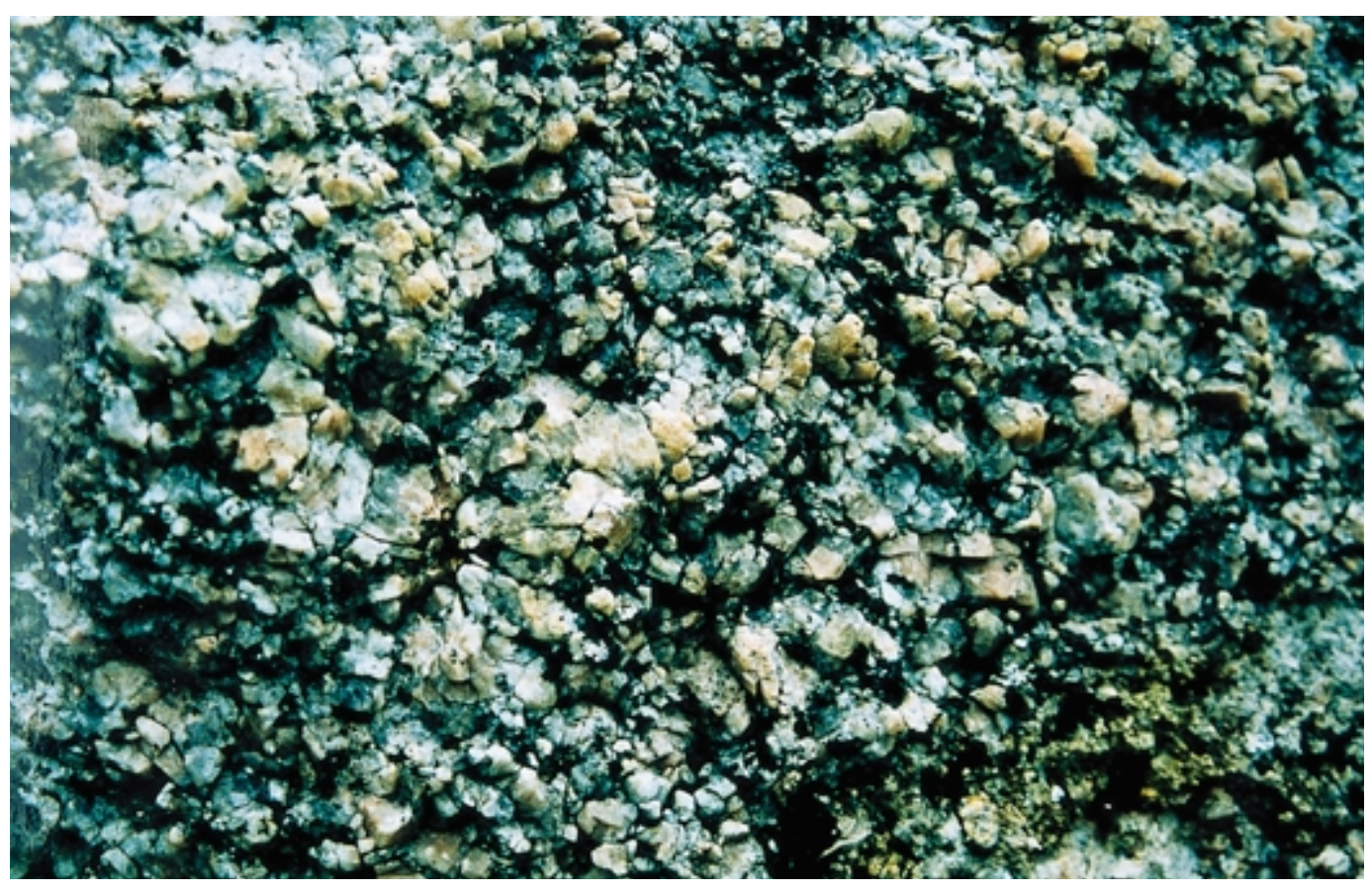

Francisco Gutiérrez

Asesor Técnico-Laboratorio.

Departamento de Análisis.

Centro de Intervención IAPH

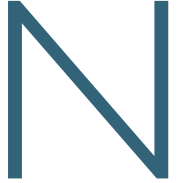

- existe una única técnica analítica capaz de realizar cualquier tipo de análisis. Dependiendo de las características físico-químicas de la muestra, unas técnicas serán más adecuadas que otras. Frecuentemente, hay que recurrir a varias técnicas para obtener un análisis lo más completo posible. En estos casos, la espectrometría infrarroja resulta especialmente útil como técnica de análisis previo para dirigir posteriores análisis.

Las técnicas instrumentales de análisis están basadas en la medida de una propiedad física o físico-química del material objeto de análisis, utilizando determinados instrumentos. En general, miden una propiedad que determina el comportamiento de los materiales en función de su composición y estructura atómica o molecular.

Dependiendo del tipo de propiedad medida podemos establecer una clasificación de las distintas técnicas instrumentales en dos grupos fundamentales:

- Técnicas espectroscópicas. Son aquellas que estudian la interacción de la materia con las radiaciones electromagnéticas. 
- Técnicas de separación. Se basan en la separación de los componentes de una mezcla y su identificación posterior. Forman parte de este grupo las técnicas cromatográficas (capa fina, gases y HPLC) y la espectrometría de masas.

En este artículo vamos a realizar una aproximación a la aplicación de las técnicas espectroscópicas, y concretamente la espectrometría infrarroja por transformada de Fourier (espectrometría FT-IR) al análisis químico de los bienes culturales.

Empecemos por considerar brevemente el fundamento teórico de esta técnica.

\section{FUNDAMENTO DE LA ESPECTROMETRÍA FT-IR}

La espectrometría FT-IR, al ser una técnica espectroscópica está basada en la interacción de la radiación electromagnética con la materia. Consideremos algunos aspectos básicos de las radiaciones electromagnéticas.

La naturaleza ondulatoria de las radiaciones electromagnéticas permite su caracterización mediante parámetros tales como: longitud de onda, $\lambda$ (distancia entre dos máximos de onda sucesivos), frecuencia, $v$ (número de ondas en un segundo) y energía asociada a la onda (E).

Longitud de onda y frecuencia están relacionados entre sí por la expresión:

$$
v=\mathrm{c} / \lambda
$$

Siendo c la velocidad de la luz en el vacío.

Sabemos que la energía no es continua, es decir, no es posible cualquier valor de energía, sino determinados valores, de acuerdo con los principios de la química cuántica. Cada uno de esos valores de energía permitidos se denominan niveles de energía. Las transiciones entre dos niveles de energía $\mathrm{E}_{2}$ y $\mathrm{E}_{1}$, siendo $E_{2}>E_{1}$, dan lugar a los fenómenos de emisión-absorción de radiación.

$\mathrm{E}_{2}$

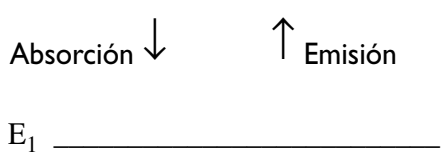

La diferencia de energía entre los dos niveles está relacionada con la frecuencia a través de la expresión:

$$
\mathrm{E}_{2}-\mathrm{E}_{1}=\mathrm{hv}
$$

Siendo h la constante de Planck

La tendencia termodinámica de cualquier sistema es ocupar siempre un estado de mínima energía, lo que supone máxima estabilidad.
Supongamos un compuesto químico, donde sus moléculas estén ocupando un nivel de energía $\mathrm{E}_{1}$. Al recibir una cantidad de energía $\mathrm{E}_{2}-\mathrm{E}_{1}$ se producirá un tránsito desde el nivel $\mathrm{E}_{1}$ al nivel $\mathrm{E}_{2}$. El proceso se denomina absorción.

Como el nivel $\mathrm{E}_{2}$ es un estado de mayor energía, la tendencia de las moléculas del compuesto será pasar al nivel $\mathrm{E}_{1}$, emitiendo el exceso de energía $\mathrm{E}_{2}-\mathrm{E}_{1}$. El fenómeno se denomina emisión.

La luz es una radiación electromagnética que se extiende desde los rayos cósmicos a las ondas térmicas y de radio. Su espectro puede dividirse en las siguientes regiones (de mayor a menor energía o de menor a mayor longitud de onda, puesto que energía y longitud de onda son magnitudes inversamente proporcionales):

- Rayos cósmicos

- Rayos gamma

- Rayos X

- Ultravioleta

- Luz visible

- Infrarrojo

- Ondas térmicas y de radio

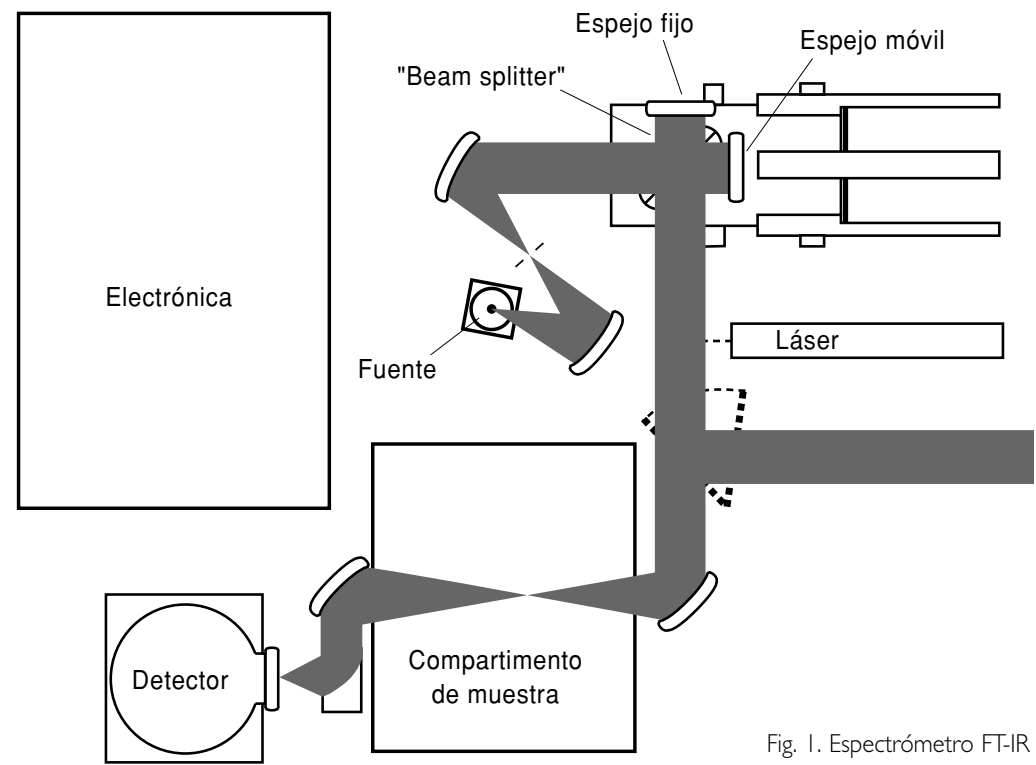

El rango espectral que define la región infrarroja es $10-12800 \mathrm{~cm}^{-1}$. Tradicionalmente, la región infrarroja se suele subdividir en tres partes:

$$
\begin{array}{lr}
\text { - Infrarrojo lejano } & 10-\quad 200 \mathrm{~cm}^{-1} \\
\text { - Infrarrojo medio } & 200-4000 \mathrm{~cm}^{-1} \\
\text { - Infrarrojo cercano } & 4000-12800 \mathrm{~cm}^{-1}
\end{array}
$$

Fundamentalmente, la región que presenta mayores aplicaciones analíticas es la que va desde los 200 a los $4000 \mathrm{~cm}^{-1}$, es decir, el denominado infrarrojo medio. Al hablar de radiación infrarroja nos vamos a referir a esta región. 
Como toda radiación lleva asociada una energía, la radiación infrarroja al incidir sobre una muestra producirá fenómenos de absorción-emisión, según hemos visto anteriormente.

La energía asociada a la radiación infrarroja es pequeña (menor que la asociada a la luz visible) y sólo es capaz afectar a los niveles vibracionales y rotacionales de las moléculas. La espectrometría FT-IR que utiliza, como fuente de excitación, la radiación infrarroja es, por tanto, una técnica de espectroscopía molecular. Permite la identificación de compuestos orgánicos y algunos inorgánicos.

Cuando sobre una muestra incide radiación infrarroja, se produce la absorción selectiva de esta radiación. Esta absorción de radiación infrarroja se manifiesta en una excitación de las moléculas de la muestra, dando lugar a vibraciones moleculares características a determinadas longitudes de onda, lo que permite la identificación del grupo funcional (agrupación de átomos en una molécula que le confiere unas propiedades químicas determinadas) y a partir de ahí caracterizar el tipo de sustancia. Una breve descripción del funcionamiento de un espectrómetro FT-IR es la siguiente:

Obra: Dolorosa. Pedro de Mena. Muestra: Manto azul. Película gomosa sobre estuco. Composición química: Resina sintética (posiblemente Polaroid)

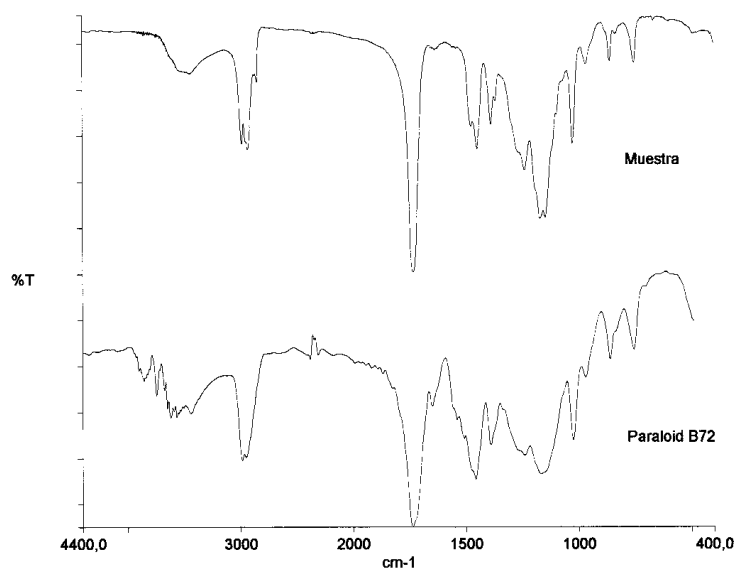

Obra: Ecce-Homo. Alonso Cano. Muestra: Barniz. Encarnadura.

Composición química: Resina natuaral terpénica (posiblemente ámbar)

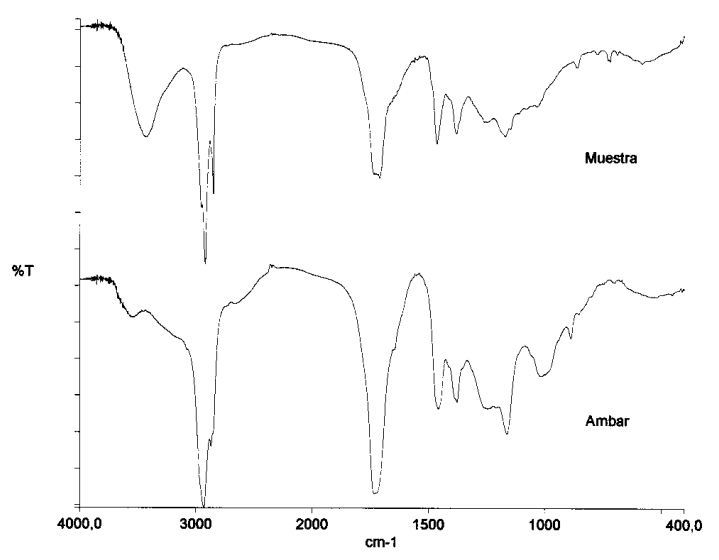

La fuente de radiación emite un haz infrarrojo que, una vez colimado, incide en el sistema óptico (interferómetro de Michelson). Este interferómetro consta de tres partes:

- el divisor del haz de rayos (beam splitter)

- un espejo fijo

- un espejo móvil

La radiación incide sobre el divisor del haz de radiación infrarroja, de modo que el $50 \%$ de la radiación incidente se dirige hacia el espejo móvil, mientras que el resto es reflejada por el espejo fijo. Una vez que ha incidido sobre ambos espejos, la radiación se recombina y llega de nuevo al divisor de haz. El 50\% de la radiación obtenida se refleja, mientras que el resto se transmite, atravesando el compartimento de la muestra y llega al detector. La diferencia de camino óptico entre las fracciones procedentes de los dos espejos hace que se produzcan interferencias constructivas y destructivas, según los haces estén en fase o en desfase, respectivamente. Cuando las interferencias sean constructivas el detector producirá una señal, mientras que cuando sean destructivas no registrará ninguna señal.

Esta señal producida por el detector se denomina interferograma y contiene información sobre la intensidad en función del tiempo. Mediante un algoritmo matemático (Transformada de Fourier) se convierte en el espectro infrarrojo, que es una representación gráfica de la intensidad de absorción de radiación frente a la frecuencia.

De la interpretación del espectro infrarrojo podrá identificarse el compuesto presente en la muestra.

\section{ÁMBITO de APLICACIÓN. SUSTANCIAS ANALIZABLES}

La espectrometría FT-IR es una técnica instrumental que permite, fundamentalmente, la identificación de materiales orgánicos. Se aplica al análisis de adhesivos, consolidantes, aglutinantes, barnices y colorantes. También se aplica en algunos casos al análisis de algunos materiales inorgánicos, como es el caso de la determinación de sulfatos, carbonatos, silicatos, nitratos, oxalatos y algunos más.

Todos estos compuestos pueden formar parte de materiales pétreos y capas pictóricas y de preparación. La espectrometría FT-IR es una técnica de aplicación general al análisis químico de los bienes culturales.

El análisis de los materiales orgánicos que forman parte de los bienes culturales es, en general, mucho más difícil que el de los materiales inorgánicos, ya que la mayoría de las veces son mezclas complejas de macromoléculas y sólo puede disponerse de cantidades de muestra muy pequeñas. Los materiales orgánicos naturales originales suelen además estar acompañados de productos de degradación, con lo cual raras veces se puede determinar con exactitud la composición y a veces hay que conformarse con la determinación del tipo de material de que se trata. 
Los compuestos orgánicos que pueden aparecer en las capas pictóricas y de preparación deben su uso a sus propiedades filmógenas (son sustancias que se aplican en estado líquido y al secar son capaces de formar capas o películas), utilizándose para la preparación de aglutinantes, adhesivos, consolidantes y barnices. Corresponden a los siguientes grupos de sustancias:

\section{Proteínas}

Son polímeros de aminoácidos unidos por enlaces peptídicos. Son las colas animales (derivados del colágeno), el huevo y la caseína (extraída de la leche). Polisacáridos

Son polímeros de monosacáridos. Los compuestos más destacados son almidón, celulosa, goma arábiga, etc.

\section{Ceras}

Según su procedencia se clasifican en:

- Ceras animales: Son ésteres de ácidos grasos saturados y alcoholes grasos. Contienen, además, pequeñas cantidades de hidrocarburos, ácidos y alcoholes libres. El ejemplo más característico es la cera de abejas.

- Ceras vegetales: Por ejemplo, la cera de carnauba que contiene derivados aromáticos.

- Ceras minerales: parafina, cera microcristalina. Están constituidas por hidrocarburos saturados derivados del petróleo.

\section{Aceites secantes}

Están compuestos por triglicéridos de ácidos grasos fundamentalmente insaturados de dieciocho carbonos. Los más utilizados son: el aceite de lino, de nueces y de adormideras.

\section{Resinas naturales}

Se emplean fundamentalmente en la formulación de barnices. Son moléculas policíclicas constituidas por polímeros del isopreno. Las más importantes son las resinas diterpénicas (colofonia, trementinas, sandaraca, copal, ámbar) y las triterpénicas (almáciga, dammar, élemi)

\section{Materiales bituminosos}

Están constituidos por hidrocarburos. Son los betunes y alquitranes.

\section{Materiales sintéticos}

Son de utilización reciente en los procesos de restauración. Están constituidos por polímeros diversos. Según el monómero de partida, se clasifican en distintos grupos: derivados de la celulosa (ésteres: acetato, propionato, acetobutirato, acetopropionato, nitrato de celulosa ; éteres: metil, etil, carboximetil e hidroximetil celulosas), derivados del almidón (dextrinas, éster y éter de almidón), derivados del caucho, organosilícicos, polietilenos, resinas vinílicas, fenólicas, cetónicas, poliésteres, acrílicas, poliamidas, epóxidos, poliuretanos, amino-resinas, poliestirenos, policloroprenos, etc. Su identificación suele ser bastante compleja.

Estos compuestos orgánicos que aparecen en las obras de arte están formados por mezclas más o menos heterogéneas. Por ejemplo, una cera, como la de abeja, está formada por cientos de compuestos diferentes (hidrocarburos, ésteres de ácidos grasos y alcoholes). Una resina como la de pino, contiene del orden de decenas de ácidos terpénicos. Una sustancia plástica, como el acetato de polivinilo, posee además de una mezcla de polímeros de diferente longitud, una serie de aditivos (conservantes, plastificantes, emulsionantes).

En el caso de mezclas, puede requerirse la separación previa de los componentes por extracción con disolventes, cromatografía preparativa, etc.

Obra: Alfombra nazarí. Muestra: Cera.

Composición química: Cera de abejas

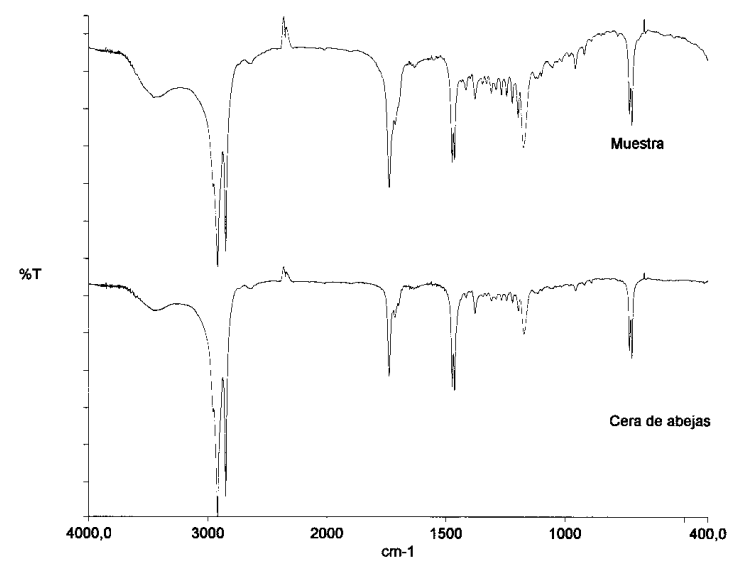

Obra: San Juan Bautista predicando en el desierto. Muestra: Estuco. (marco) Composición química: Yeso, carbonato cálcico y proteína

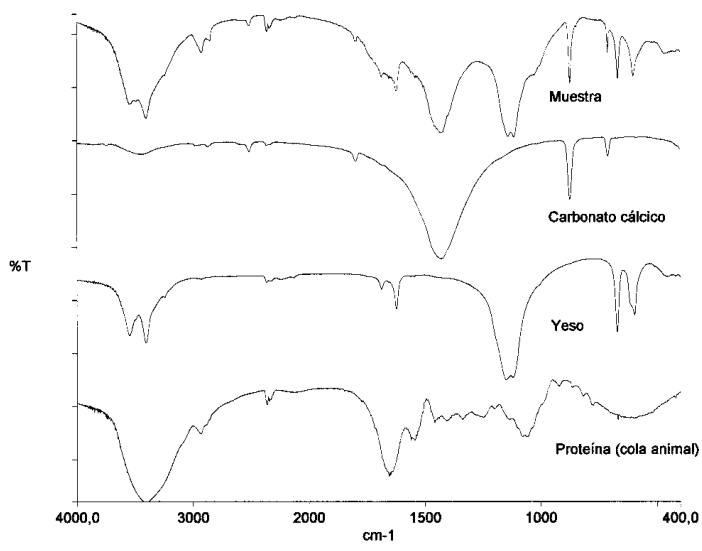


눔
Hay una serie de factores que van a complicar aún más el análisis:

- Envejecimiento de los materiales. Con el transcurso del tiempo se producen una serie de reacciones químicas entre los materiales constitutivos para dar lugar a la formación de una serie de productos que difieren de los inicialmente aplicados.

- Mezclas de mezclas: Si una resina es una mezcla compleja de compuestos, pensemos la complejidad de un sistema formado por una cera con una resina.

- Sistemas multicapa, habituales en pintura y que exigen la separación física de las diferentes capas.

- Tamaño de las muestras. La cantidad de muestra disponible para analizar suele ser muy pequeña, con lo que deben usarse técnicas de alta sensibilidad.

- Productos añadidos durante las diferentes intervenciones y que dificultan el análisis de los materiales originales.

Obra: Virgen de la amargura. Muestra: Pasta de relleno Composición química: Yeso y proteína

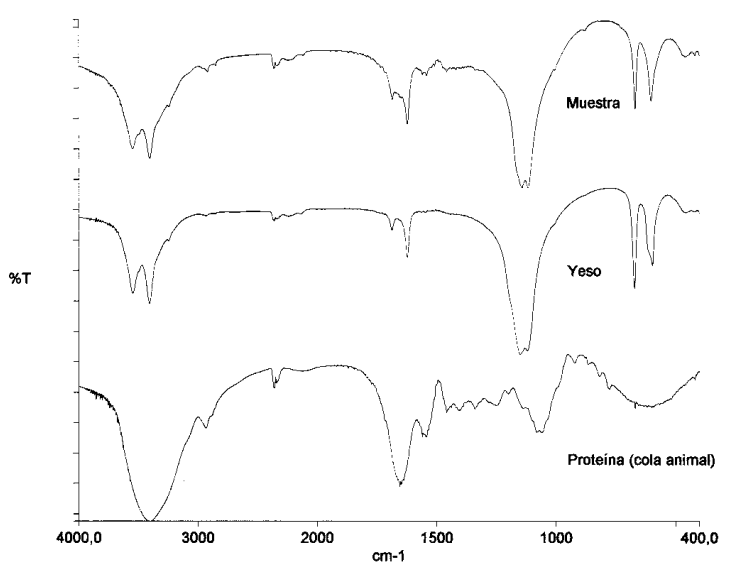

Analizar materiales tan complejos requiere la utilización de patrones, sustancias de referencia de composición conocida, con las que comparar el espectro de la muestra.

\section{Preparación de muestras}

Existen diversos procedimientos para la preparación de muestras para análisis por espectrometría infrarroja. El más utilizado, en el caso de muestras sólidas (las más frecuentes) es el de la preparación de pastillas de bromuro potásico.

El procedimiento es muy simple y consiste en moler unos pocos miligramos de muestra con bromuro potásico en un mortero de ágata. Se utiliza bromuro potásico por tratarse de un compuesto transparente a la radiación infrarroja, por lo que no interfiere en la identificación de los componentes de la muestra.

La mezcla obtenida se prensa y la pastilla obtenida se introduce en el compartimento de muestras para la obtención del espectro.

\section{Casos prácticos}

Para ilustrar la aplicación de esta técnica al análisis químico de los bienes culturales, se consideran algunos casos prácticos analizados en el Laboratorio de Química del IAPH.

En cada caso el espectro de la muestra se compara con el de los patrones correspondientes.
LOW M. J. D., and BAER N.S. Application of infrared Fourier transform spectroscopy to problems in conservation. I. General principles. Studies in Conservation 22 (1977) I 16- 128.

GÓMEZ GONZÁLEZ Ma L. Examen científico aplicado a la conservación de obras de arte. Ministerio de Cultura 1994.
Curso de técnicas de diagnóstico aplicadas a la conservación de los bienes muebles. Granada 1996.

C. J. SERNA. Espectroscopía IR, visible y UV. Introducción a la ciencia de materiales: técnicas de preparación y caracterización. CSIC 1993. Pág. 67|-70|.

PARRA, E. Análisis de materiales orgánicos. Técnicas y aplicaciones. 